\title{
Evaluation of Two Phenotypic Methods for the Detection of Plasmid-Mediated AmpC $\beta$-Lactamases among Enterobacteriaceae Isolates
}

\author{
Ronni Mol P1,2 Khalid Mubarak Bindayna ${ }^{2}$ Ganesan Shanthi ${ }^{1}$ \\ ${ }^{1}$ Division of Microbiology (Medicine), Rajah Muthaiah Medical \\ College, Annamalai University, Chidambaram, Tamil Nadu, India \\ ${ }^{2}$ Department of Microbiology, Immunology and Infectious Diseases, \\ College of Medicine and Medical Sciences, Arabian Gulf University, \\ Address for correspondence Ronni Mol P, MBBS, MD, PhD Research \\ Scholar,Division of Microbiology (Medicine), Rajah Muthaiah Medical \\ College, Annamalai University, Chidambaram 608002, Tamil Nadu, \\ India (e-mail: ronnimj@agu.edu.bh).
} Manama, Kingdom of Bahrain

J Lab Physicians 2021;13:151-155.

\begin{abstract}
Keywords

- Enterobacteriaceae

- AmpC $\beta$-lactamases

- inhibitor-based assay

- disk approximation test

- multiplex PCR

Objectives AmpC $\beta$-lactamases are cephalosporinases that confer resistance to cephalothin, cefazolin, cefoxitin, penicillin, and $\beta$-lactamase inhibitor- $\beta$-lactam combinations. Even though the $A m p C$ resistance is reported, but the accurate occurrence of AmpC $\beta$-lactamases in Enterobacteriaceae members is still unknown. Techniques to identify AmpC producers are still evolving but not yet optimized for the clinical laboratory. Here we aimed to compare the test performance of two different phenotypic methods, that is inhibitor-based assay using boronic acid and disk approximation test for AmpC detection in Enterobacteriaceae isolates from a tertiary hospital microbiology laboratory.

Materials and Methods The study includes 137 nonrepeat Enterobacteriaceae strains. Bacterial isolates, that yielded a zone diameter of less than $18 \mathrm{~mm}$ for cefoxitin by disk diffusion method were considered potential AmpC producers and further confirmed by phenotype methods-inhibitor-based assay using boronic acid and disk approximation test. A multiplex polymerase chain reaction was used to detect the most common plasmid-mediated AmpC genes: ACC, FOX, MOX, DHA, CIT, and EBC.

Results Of the 137 clinical isolates, 58 (42.33\%) were cefoxitin resistant, while 53.4 and $18.9 \%$ of the cefoxitin-resistant isolates were positive by inhibitor-based assay and disk approximation test. Multiplex PCR detected 42 (30.6\%) isolates with AmpC genes. Of the 42 isolates, the inhibitor-based assay detected 25 (59.5\%) isolates, while the disk approximation test detected nine (21.4\%) isolates.

Conclusion Our findings suggest that inhibitor-based assay using boronic acid can be used for the detection of the isolates that harbor AmpC $\beta$-lactamases. This method is cost-effective, simple to perform, and easy to interpret. Thus AmpC detection as a routine in clinical laboratories can help in appropriate therapeutic intervention and improved infection control.
\end{abstract}

published online June 15, 2021
DOI https://doi.org/

10.1055/s-0041-1729472 ISSN 0974-2727 (c) 2021. The Indian Association of Laboratory Physicians.

This is an open access article published by Thieme under the terms of the Creative Commons Attribution-NonDerivative-NonCommercial-License, permitting copying and reproduction so long as the original work is given appropriate credit. Contents may not be used for commercial purposes, or adapted, remixed, transformed or built upon. (https://creativecommons.org/licenses/by-nc-nd/4.0/). Thieme Medical and Scientific Publishers Pvt. Ltd. A-12, 2nd Floor, Sector 2, Noida-201301 UP, India 


\section{Background}

AmpC $\beta$-lactamases are cephalosporinases that confer resistance to cephalothin, cefazolin, cefoxitin, penicillin, and $\beta$-lactamase inhibitor- $\beta$-lactam combinations. ${ }^{1}$ Under Ambler classification scheme, AmpC $\beta$-lactamases are class $C$ enzymes, which utilize serine for beta-lactam hydrolysis. ${ }^{2}$ AmpC $\beta$-lactamase resistance mechanisms can be: (1) inducible resistance via chromosomally encoded AmpC genes which is present in Citrobacter freundii, Enterobacter cloacae, etc., (2) non-inducible chromosomal resistance due to promoter and/or attenuator mutations seen in Escherichia coli, Shigella species, and (3) plasmid-mediated resistance in Klebsiella pneumoniae, E. coli, Salmonella species, etc. ${ }^{3}$

Even though the AmpC resistance is reported, but the accurate occurrence of AmpC $\beta$-lactamases in Enterobacteriaceae members is still unknown. ${ }^{4}$ In Enterobacteriaceae, cefoxitin resistance is used for screening of AmpC $\beta$-lactamase producers. Its resistance may also be due to alterations to outer membrane permeability. ${ }^{5}$ Techniques to identify AmpC producers are still evolving but not yet optimized for clinical laboratory. ${ }^{6}$ Disk-based assays using cloxacillin and inhibitors (boronic acid [BA] compounds), cefoxitin-cloxacillin double disk synergy, AmpC disk tests, disk approximation tests, etc. have been developed for detection of AmpC-producing $\beta$-lactamase isolates in Enterobacteriaceae. ${ }^{5,6}$ Molecular tests are also available but their use is restricted to research settings. ${ }^{3}$ The Clinical and Laboratory Standards Institute(CLSI) guidelines 2019 recommended criteria for $\mathrm{AmpC}$ resistance detection do not exist.

However, surveillance and monitoring activity is significantly important in this epoch of multidrug resistance as failure in antimicrobial resistant mechanisms detection may result in the spread of resistant pathogens and ultimately, complicating the clinical outcome. ${ }^{5}$ Well-designed studies on diagnostic techniques for the detection of AmpC $\beta$-lactamases, that is easy-to-perform and provides reliable results in a short time, and suitable for treatment recommendations for AmpC-producers are needed.?

This study aimed to compare the test performance of two different phenotypic methods that is inhibitor-based assay using BA and disk approximation test for AmpC detection in Enterobacteriaceae isolates from a tertiary hospital microbiology laboratory.

\section{Material and Methods}

\section{Bacterial Isolate Collection and Identification}

The study was based on laboratory surveillance from July 2018 to February 2019. The analysis includes 137 nonrepeat Enterobacteriaceae strains (60 E. coli, $60 \mathrm{~K}$. pneumoniae, 10 Proteus species, 5 Citrobacter koseri, 1 Salmonella typhi, and 1 Enterobacter species samples). The clinical isolates were identified by their colony morphology, Gram staining characteristics, and standard biochemical tests. ${ }^{8}$ We evaluated antimicrobial susceptibility by Kirby Bauer method as per CLSI (formerly NCCLS) guidelines, 2018.

\section{AmpC $\beta$-lactamase Detection}

Bacterial isolates that yielded a zone diameter of less than $18 \mathrm{~mm}$ for cefoxitin by disk diffusion method were considered potential AmpC producers ${ }^{9}$ which was further confirmed by phenotype methods-inhibitor-based assay using BA and disk approximation test.

Inhibitor-based assay: Mueller Hinton agar (MHA) plates were inoculated with the bacterial isolate. Cefoxitin-BA disks were prepared as per Coudron. ${ }^{10}$ Cefoxitin and cefoxitin with BA disks were placed on the inoculated MHA plates and incubated overnight at $37^{\circ} \mathrm{C}$. An isolate that demonstrated a zone diameter of $5 \mathrm{~mm}$ or more in the presence of cefoxitin with $\mathrm{BA}$ in comparison with cefoxitin alone was considered an AmpC producer ( - Fig. 1).

Disk approximation test: MHA plates were inoculated with the study isolate. A ceftazidime disk $(30 \mu \mathrm{g})$ was placed at the center of the inoculated plate. Imipenem $(10 \mu \mathrm{g})$, cefoxitin $(30 \mu \mathrm{g})$, and amoxicillin/clavulanate $(20 / 10 \mu \mathrm{g})$ disks were then placed at a distance of $20 \mathrm{~mm}$ from the ceftazidime disk. The inoculated plates were incubated overnight at $37^{\circ} \mathrm{C}$. After overnight incubation, if the isolate demonstrates an obvious blunting or flattening of the zone of inhibition between the ceftazidime disk and the inducing substrates (imipenem, cefoxitin, and amoxicillin/clavulanate disk) then the isolate was considered as an AmpC producer ${ }^{11}$ (-Fig. 2).

Molecular characterization of AmpC $\beta$-lactamase: Multiplex polymerase chain reaction (PCR) was used to detect the most common plasmid-mediated AmpC genes: ACC, FOX, MOX, DHA, CIT, and EBC. ${ }^{11}$

The protocol for multiplex was as follows: for the detection of MOX gene, 5GCTGCTCAAGGAGCACAGGAT-3 was used

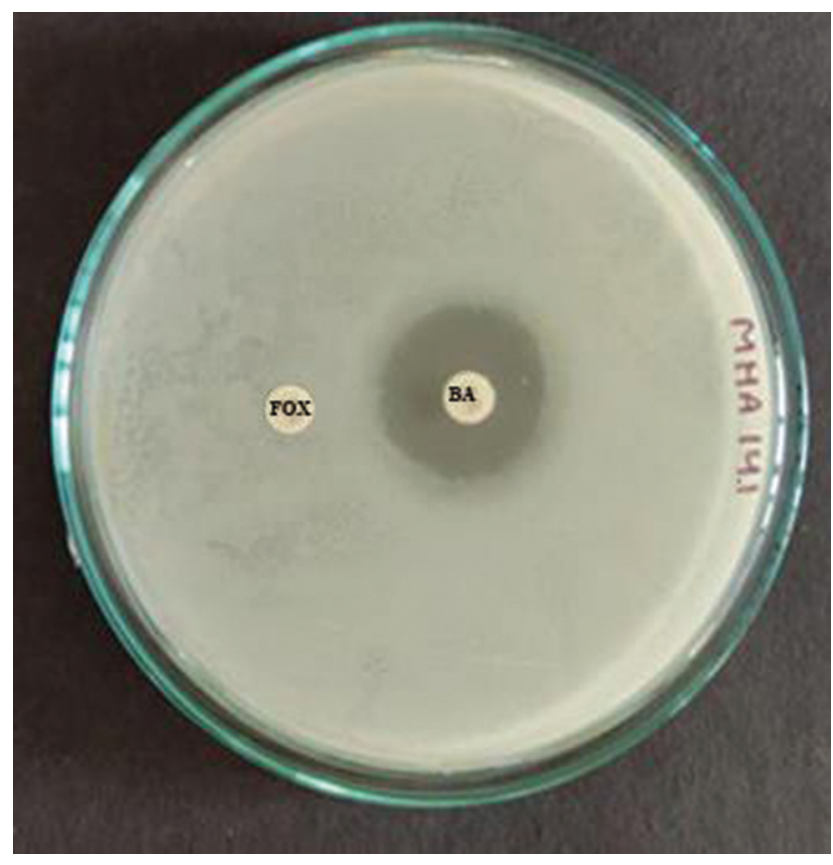

Fig. 1 Representation of inhibitor-based assay. A pure AmpC-producing isolate showing cefoxitin (FOX) zone enhancement $(\geq 5 \mathrm{~mm})$ with the addition of BA. BA, boronic acid. 


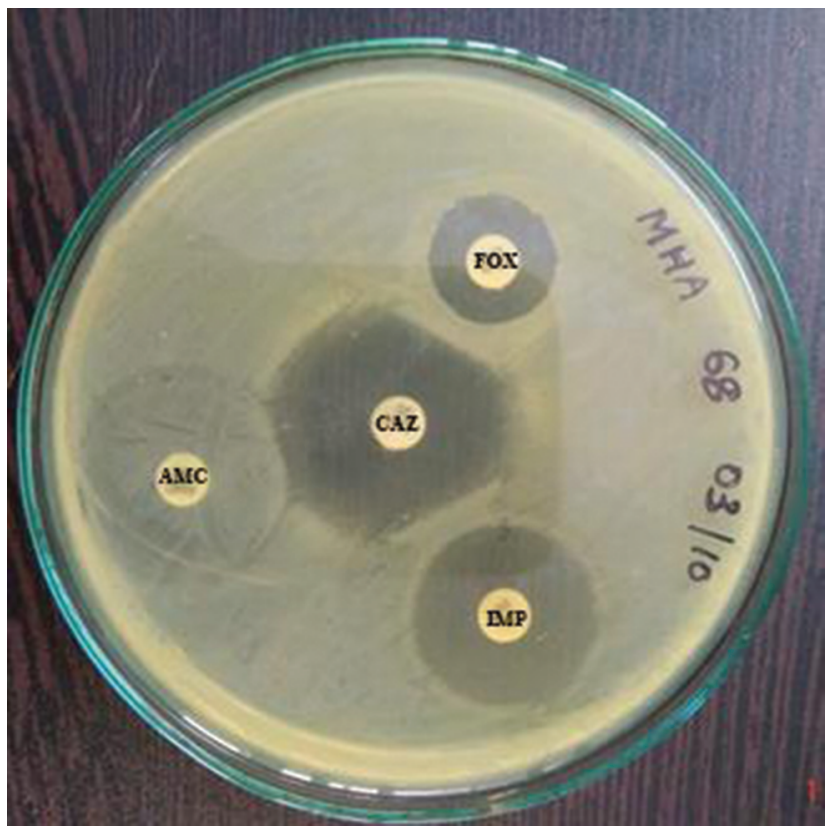

Fig. 2 Representation of disk approximation test. Flattening of the zone of ceftazidime toward imipenem disk and cefoxitin disk showing AmpC producer. IMP, imipenem $(10 \mu \mathrm{g})$; FOX, cefoxitin $(30 \mu \mathrm{g})$; CAZ, ceftazidime $(10 \mu \mathrm{g})$; AMC, amoxicillin-clavulanate $(20 / 10 \mu \mathrm{g})$.

as forward primer and 5-CACATTGACATAGGTGTGGTGC-3 was used as the reverse primer, expected amplicon size 520 bp. For the detection of CIT gene, 5-TGGCCAGAACTGACAGGCAAA-3 was used as forward primer and 5-TTTCTCCTGAACGTGGCTGGC-3 was used as the reverse primer, expected amplicon size $462 \mathrm{bp}$. For the detection of DHA gene, 5-AACTTTCACAGGTGTGCTGGGT-3 was used as forward primer and 5-CCGTACGCATACTGGCTTTGC-3 was used as the reverse primer, expected amplicon size $405 \mathrm{bp}$. For the detection of ACC gene, 5-AACAGCCTCAGCAGCCGGTTA-3 was used as forward primer and 5-TTCGCCGCAATCATCCCTAGC-3 was used as the reverse primer, expected amplicon size 346 bp. For the detection of EBC gene, 5-TCGGTAAAGCCGATGTTGCGG-3 was used as forward primer and 5-CTTCCACTGCGGCTGCCAGTT-3 was used as the reverse primer, expected amplicon size $302 \mathrm{bp}$. For the detection of FOX gene, 5-AACATGGGGTATCAGGGAGATG-3 was used as forward primer and 5-CAAAGCGCGTAACCGGATTGG-3 was used as the reverse primer, expected amplicon size $190 \mathrm{bp}$.

DNA extraction was by Modified Proteinase K method. ${ }^{12}$ For PCR assays, $2-\mu \mathrm{L}$ cDNA was added to $23-\mu \mathrm{L}$ master mixture of PCR reagents. The reaction was programmed with initial denaturation step at $94^{\circ} \mathrm{C}$ for 3 minutes; followed by 25 cycles of DNA denaturation at $94^{\circ} \mathrm{C}$ for 30 seconds, primer annealing at $64^{\circ} \mathrm{C}$ for 30 seconds, primer extension at $72^{\circ} \mathrm{C}$ for 1 minute; and a final extension step at $72^{\circ} \mathrm{C}$ for 7 minutes ${ }^{6}$ (Department of Molecular Biology and Immunology, MMNGH Institute of Dental Sciences and Research Centre, Belgaum, for Multiplex PCR). Amplified products were subjected to electrophoresis through 3\% Agarose gel. $16 \mu \mathrm{L}$ of each amplified product was loaded into each well. The gel was visualized under UV light illuminator and analyzed using Gel Documentation System (Major Science, California, United States). Negative control used was PCR mix with distilled water and a 100 base-pair DNA ladder was used as the size reference (-Fig. $\mathbf{3}$ ).

Statistical analysis: Statistical Package for the Social Science version 20 (IBM, Armonk, New York, United States) was employed to obtain descriptive data.

\section{Results}

Antimicrobial susceptibility testing: The clinical isolates showed resistance to multiple antimicrobial drugs. All our isolates showed complete resistance to ampicillin (100\%). This was found to coexist with resistance to two or more antimicrobials that is cotrimoxazole (47\%), norfloxacin (30.6\%), ciprofloxacin $(23.1 \%)$, and gentamicin (15.5\%). Least resistance was observed with amikacin (1.1\%) and piperacillin/tazobactam (2.2\%).

Phenotypic detection of AmpC $\beta$-lactamases: Among 58 (42.3\%) cefoxitin-resistant isolates, 53.4 and $18.9 \%$ of the isolates were positive by inhibitor-based assay and disk approximation test ( $\mathbf{- T a b l e ~} \mathbf{1}$ ).

PCR detection of AmpC genes: Overall, of 137 Enterobacteriaceae members, 42 (30.6\%) isolates were positive for AmpC gene subtypes (-Table 2).

Comparison of phenotypic test results with PCR detection of AmpC genes: Of the 42 isolates with Amp genes detected by multiplex PCR, inhibitor base assay detected $25(59.5 \%)$ isolates, while disk approximation test detected 9 (21.4\%) isolates. - Table 3 shows the statistical analysis of inhibitor-based assay and disk approximation test in comparison with gold standard PCR assay.

\section{Discussion}

Plasmid-mediated AmpC resistance pose a big challenge to infection control as the AmpC genes are expressed in higher amounts and are highly transmissible to other

Table 1 Detection of AmpC producers by phenotypic methods

\begin{tabular}{|l|l|l|l|}
\hline Isolates & Cefoxitin screening & Inhibitor-based assay & Disk approximation test \\
\hline Escherichia coli $(n=60)$ & $28(46.6 \%)$ & $14(23.3 \%)$ & $5(8.3 \%)$ \\
\hline Klebsiella pneumoniae $(n=60)$ & $23(38.3 \%)$ & $13(21.6 \%)$ & $5(8.3 \%)$ \\
\hline Proteus species $(n=10)$ & $4(40 \%)$ & $3(30 \%)$ & $1(10 \%)$ \\
\hline Citrobacter koseri $(n=5)$ & $2(40 \%)$ & $1(20 \%)$ & $0(0 \%)$ \\
\hline Salmonella typhi $(n=1)$ & $0(0 \%)$ & $0(0 \%)$ & $0(0 \%)$ \\
\hline Enterobacter species $(n=1)$ & $1(100 \%)$ & $0(0 \%)$ & $0(0 \%)$ \\
\hline Total $(n=137)$ & $58(42.3 \%)$ & $31(22.6 \%)$ & $11(8 \%)$ \\
\hline
\end{tabular}


bacterial species. ${ }^{13}$ Worldwide prevalence of AmpC resistance is unknown, due to the limited number of surveillance studies and lack of laboratory diagnostic techniques in accurately detecting this resistance mechanism. ${ }^{14}$ Detection of AmpC $\beta$-lactamases is clinically important so as to avoid therapeutic failures and nosocomial outbreaks. ${ }^{4}$ Therapeutic options for AmpC producers are limited due to resistance to most of the $\beta$-lactam drugs except for cefepime and carbapenem. ${ }^{15}$

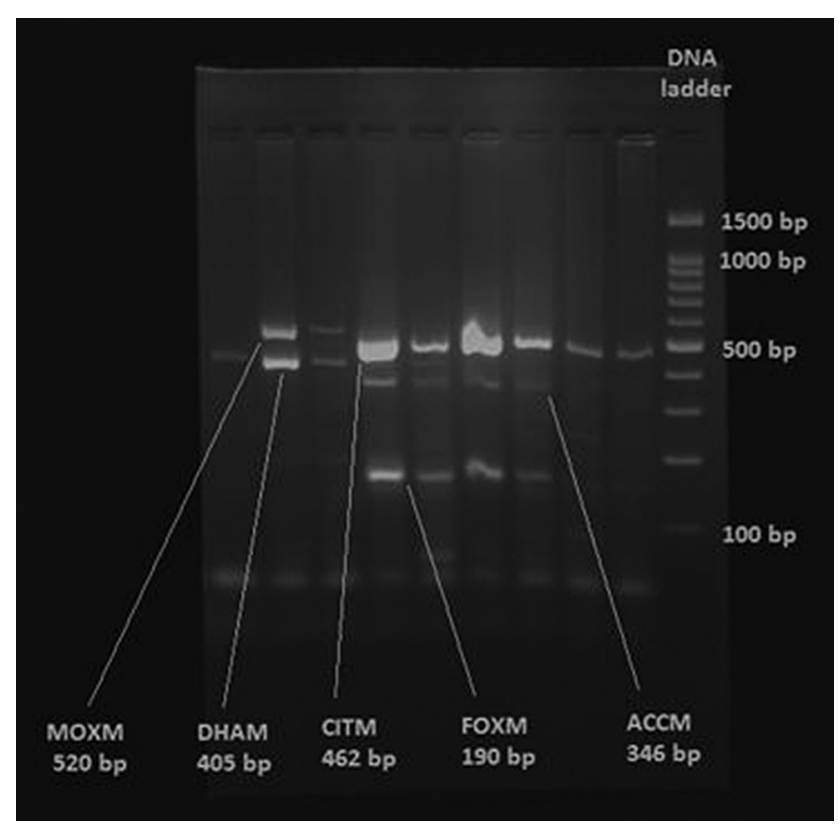

Fig. 3 AmpC genes by multiplex PCR. PCR, polymerase chain reaction.

Table 2 Distribution of AmpC genes among Enterobacteriaceae isolates

\begin{tabular}{|l|l|}
\hline Isolates & AmpC genotype positive \\
\hline Escherichia coli $(n=60)$ & 17 \\
\hline Klebsiella pneumoniae $(n=60)$ & 19 \\
\hline Proteus species $(n=10)$ & 3 \\
\hline Citrobacter koseri $(n=5)$ & 2 \\
\hline Enterobacter species $(n=1)$ & 1 \\
\hline Total $n=137$ & 42 \\
\hline
\end{tabular}

In the present study, the clinical isolates showed complete resistance to ampicillin (100\%) followed by cotrimoxazole (47\%), norfloxacin (30.6\%), ciprofloxacin (23.1\%), and gentamicin (15.5\%). Least resistance was observed with amikacin (1.1\%) and piperacillin/tazobactum (2.2\%). Similar antimicrobial resistance patterns have been observed among Indian Enterobacteriaceae clinical isolates, ${ }^{16,17}$ whereas a study from Romania has reported high antimicrobial resistance against cotrimoxazole (74\%), fluoroquinolones (49\%), and penicillin (44\%). Another study from Iraq has reported high MDR rate with $\beta$-lactams, aminoglycosides, and fluoroquinolones among Enterobacteriaceae.$^{18}$ Studies in different parts of the world have shown different patterns of antimicrobial resistance. This may be due to inappropriate ignorance and overuse of antibiotics, inappropriate infection control, and lack of awareness of the clinical outcome of multidrug-resistant bacterial infections. ${ }^{16}$

The present study demonstrated that among 58 cefoxitin-resistant isolates, 42 (72.4\%) were found to possess AmpC genes by PCR. Similar results were reported by Yilmaz et $\mathrm{l}^{19}$ and Helmy and Wasfi. ${ }^{20}$ Not all cefoxitin-resistant isolates were AmpC producers. Cefoxitin resistance can be due to the presence of other antimicrobial-resistant mechanisms like extended spectrum beta lactamases, metallo beta lactamases, and mutation of porin channels. Cefoxitin also acts as a substrate to active efflux pumps in clinical strains. ${ }^{20}$

Phenotypic methods are unable to differentiate chromosomal and plasmid AmpC $\beta$-lactamases. Plasmid AmpC genes are detected by PCR analysis. But the molecular test is expensive and not available for routine use in all the clinical laboratories. ${ }^{1}$ Hence there is a practical need for a simple and cost effective assay to detect plasmid AmpC $\beta$-lactamases. Our study compared two phenotypic methods and observed 53.4 and $18.9 \%$ of the cefoxitin-resistant isolates positive by inhibitor-based assay and disk approximation test. Plasmid AmpC genes were detected in 25 (59.5\%) of the 31 inhibitor-based assay positive isolates and 9 (21.4\%) of the 11 disk approximation test positive isolates by molecular analysis. Based on these findings, the inhibitor-based assay exhibited $58 \%$ sensitivity and $60 \%$ specificity and disk approximation test exhibited $21 \%$ sensitivity and $87 \%$ specificity.

A study from the United States has noted $58 \%$ of the boronic acid disk test positive isolates gave positive result using multiplex PCR. ${ }^{21}$ In contrast, Yilmaz et al reported the presence of

Table 3 Statistical analysis of phenotypic AmpC detection tests

\begin{tabular}{|l|l|l|l|l|}
\hline \multirow{2}{*}{ Statistic } & \multicolumn{2}{|c|}{ Inhibitor-based assay } & \multicolumn{2}{c|}{ Disk approximation test } \\
\cline { 2 - 5 } & Value & $95 \% \mathrm{Cl}$ & Value & $95 \% \mathrm{Cl}$ \\
\hline Sensitivity & $58.14 \%$ & $42.13-72.99 \%$ & $21.43 \%$ & $10.30-36.81 \%$ \\
\hline Specificity & $60.00 \%$ & $32.29-83.66 \%$ & $87.50 \%$ & $61.65-98.45 \%$ \\
\hline Positive likelihood ratio & 1.45 & $0.74-2.84$ & 1.71 & $0.41-7.09$ \\
\hline Negative likelihood ratio & 0.70 & $0.41-1.20$ & 0.90 & $0.70-1.15$ \\
\hline Positive predictive value & $80.65 \%$ & $68.08-89.06 \%$ & $81.82 \%$ & $52.10-94.90 \%$ \\
\hline Negative predictive value & $33.33 \%$ & $22.51-46.25 \%$ & $29.79 \%$ & $24.96-35.11 \%$ \\
\hline Accuracy & $58.62 \%$ & $44.93-71.40 \%$ & $39.66 \%$ & $27.05-53.36 \%$ \\
\hline
\end{tabular}

Abbreviation: $\mathrm{Cl}$, confidence interval. 
AmpC genes in $22 \%$ of the boronic acid positive isolates and the inhibitor-based assay revealed $100 \%$ sensitivity and $66 \%$ specificity. ${ }^{19}$ A study from Egypt reported $100 \%$ positivity among 50/148 (33.8\%) AmpC isolates, by disk approximation test which was inconsistent with our observation. ${ }^{11}$

False positive phenotypic test results encountered in our study may be due to the presence of unknown AmpC genes or the inability of the phenotypic methods to differentiate chromosomal and plasmid AmpC genes. On the other hand, the reason for the false negative results is the ineffective phenotypic AmpC gene expression. ${ }^{20}$ The difference between the phenotype methods and the molecular test results can be explained by the presence of chromosomal AmpCs or porin mutations. ${ }^{19}$

\section{Conclusion}

Our findings suggest that inhibitor-based assay using BA is a practical and efficient method for the identification of AmpC producers. This method is cost effective, simple to perform, and easy to interpret. Therefore, inhibitor-based assay using $\mathrm{BA}$ can be used for the detection of the isolates that harbor AmpC enzymes in the clinical laboratory where multiplex PCR is not affordable and thus help in therapeutic intervention, improved infection control and prevent the dissemination of antimicrobial resistance.

Availability of data and materials: All data generated or analyzed during this study are included in this article.

\section{Financial Disclosure}

None.

\section{Ethical Approval}

This research is approved by Annamalai Institutional Review Board.

\section{Authors' Contribution}

R.M.P. contributed towards concept, design, literature search, experimental studies, data acquisition, data analysis, statistical analysis, and manuscript preparation. G.S. did the data analysis, manuscript editing, and manuscript review. K.M.B. contributed towards data analysis, manuscript editing, and manuscript review.

\section{Conflict of Interest}

None declared.

\section{References}

1 Jacoby GA. AmpC beta-lactamases. Clin Microbiol Rev 2009;22(1):161-182, Table of Contents. Epub 2009/01/13

2 Bush K, Jacoby GA. Updated functional classification of beta-lactamases. Antimicrob Agents Chemother 2010;54(3):969-976

3 Tamma PD, Doi Y, Bonomo RA, Johnson JK, Simner PJ; Group ARL; Antibacterial Resistance Leadership Group. A Primer on AmpC $\beta$-lactamases: necessary knowledge for an increasingly multidrug-resistant world. Clin Infect Dis 2019;69(8):1446-1455

4 Handa D, Pandey A, Asthana AK, Rawat A, Handa S, Thakuria B. Evaluation of phenotypic tests for the detection of AmpC beta-lactamase in clinical isolates of Escherichia coli. Indian J Pathol Microbiol 2013;56(2):135-138
5 Mohd Khari FI, Karunakaran R, Rosli R, Tee Tay S. Genotypic and phenotypic detection of AmpC $\beta$-lactamases in Enterobacter spp. isolated from a teaching hospital in Malaysia. PLoS One 2016;11(3):e0150643

6 Gupta G, Tak V, Mathur P. Detection of AmpC lactamases in gram-negative bacteria. J Lab Physicians 2014;6(1):1-6

7 Correa-Martínez CL, Idelevich EA, Sparbier K, Kostrzewa M, Becker K. Rapid detection of extended-spectrum $\beta$-lactamases (ESBL) and AmpC $\beta$-lactamases in Enterobacterales: development of a screening panel using the MALDI-TOF MS-based direct-on-target microdroplet growth assay. Front Microbiol 2019;10(13):13

8 Yadav KK, Adhikari N, Khadka R, Pant AD, Shah B. Multidrug resistant Enterobacteriaceae and extended spectrum $\beta$-lactamase producing Escherichia coli: a cross-sectional study in National Kidney Center, Nepal. Antimicrob Resist Infect Control 2015;4(1):42

9 Pal N, Hooja S, Sharma R, Maheshwari RK. Phenotypic detection and antibiogram of $\beta$-lactamase-producing Proteus species in a Tertiary Care Hospital, India. Ann Med Health Sci Res 2016;6(5):267-273

10 Coudron PE. Inhibitor-based methods for detection of plasmid-mediated AmpC beta-lactamases in Klebsiella spp., Escherichia coli, and Proteus mirabilis. J Clin Microbiol 2005;43(8):4163-4167

11 El-Hady SA, Adel LA. Occurrence and detection of AmpC $\beta$-lactamases among Enterobacteriaceae isolates from patients at Ain Shams University Hospital. Egypt J Med Hum Genet 2015;16(3):239-244

12 Vanpelt E, Belkum AV, Hays JP. Principles and Technical Aspects of PCR Amplification. Netherlands:Springer; 2008

13 Marchese A, Arlet G, Schito GC, Lagrange PH, Philippon A. Characterization of FOX-3, an AmpC-type plasmid-mediated beta-lactamase from an Italian isolate of Klebsiella oxytoca. Antimicrob Agents Chemother 1998;42(2):464-467

14 Thomson KS. Controversies about extended-spectrum and AmpC beta-lactamases. Emerg Infect Dis 2001;7(2):333-336

15 Pérez-Pérez FJ, Hanson ND. Detection of plasmid-mediated AmpC beta-lactamase genes in clinical isolates by using multiplex PCR. J Clin Microbiol 2002;40(6):2153-2162

16 Hariharan P, Bharani T, Franklyne JS, Biswas P, Solanki SS, Paul-Satyaseela M. Antibiotic susceptibility pattern of Enterobacteriaceae and non-fermenter gram-negative clinical isolates of microbial resource orchid. J Nat Sci Biol Med 2015;6(1):198-201

17 Sarma JB, Bhattacharya PK, Kalita D, Rajbangshi M. Multidrug-resistant Enterobacteriaceae including metallo- $\beta$-lactamase producers are predominant pathogens of healthcare-associated infections in an Indian teaching hospital. Indian J Med Microbiol 2011;29(1):22-27

18 Lazm AM, Al-Allak MH, Al-Asskar JA, Al-Dahmoshi HOM, Al-Khafaji NSK. Antibiotics resistance patterns among Enterobacteriaceae isolated from different clinical samples. Drug Invention Today 2019;12(5):938-942

19 Yilmaz NO, Agus N, Bozcal E, Oner O, Uzel A. Detection of plasmid-mediated AmpC $\beta$-lactamase in Escherichia coli and Klebsiella pneumoniae. Indian J Med Microbiol 2013;31(1):53-59

20 Helmy MM, Wasfi R. Phenotypic and molecular characterization of plasmid mediated AmpC $\beta$-lactamases among Escherichia coli, Klebsiella spp., and Proteus mirabilis isolated from urinary tract infections in Egyptian hospitals. BioMed Res Int 2014;2014:171548

21 Tenover FC, Emery SL, Spiegel CA, et al. Identification of plasmid-mediated AmpC beta-lactamases in Escherichia coli, Klebsiella spp., and proteus species can potentially improve reporting of cephalosporin susceptibility testing results. J Clin Microbiol 2009;47(2):294-299 